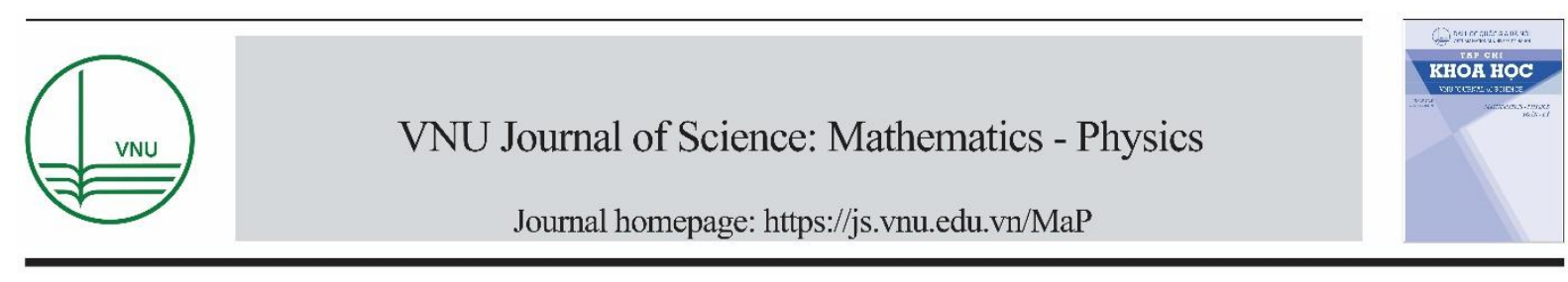

Original Article

\title{
Robust Stability of Implicit Dynamic Equations on Time Scales
}

\author{
Nguyen Thu Ha* \\ Electric Power University, 235 Hoang Quoc Viet, Cau Giay, Hanoi, Vietnam
}

Received 21 October 2019

Accepted 30 October 2019

\begin{abstract}
This paper studies the robust stability of implicit dynamic equations on time scales, which is a general form of differential algebraic equations and implicit difference equations. The paper discusses the reservation of exponential stability of these equations under small Lipschitz perturbations. Keywords: Uniform stability, time scales, implicit dynamic equations, Lipschitz perturbations.
\end{abstract}

\section{Introduction}

In lots of applications there is a frequently arising question, namely how robust is a characteristic qualitative property of a system (e.g., the stability) when the system comes under the effect of uncertain perturbations. The designer wants to have operation systems working stably under small perturbations. Therefore, the investigation which conditions ensures robust stability play an important role both in theory and practice. The classical problem studied the preservation of exponential stability of the system $y^{\prime}(t)=B y(t)+h(t, y)$, where $h(t, y)=o(y)$ as $y \rightarrow 0$ or $h(t, y)$ is Lipschitz continuous. Next, this problem develops for the difference equation $y(n+1)=B y(n)+h(n, y(n))$. To unify these results, Tien and $\mathrm{Du}$ in [1] have considered the perturbed dynamic equation on time scale $\mathbf{T}$ $y^{\Delta}(t)=B(t) y(t)+q(t), \quad t \geq a, t \in \mathbf{T}$. The aim of this paper is to continue studying the above problems. We desire to study the robust stability of time-varying systems of implicit dynamic equations (IDEs) in a general context. That is the implicit dynamic equation of the form

$$
A_{\sigma}(t) y^{\Delta}(t)=B(t) y(t), \quad t \geq a, t \in \mathbf{T},
$$

\footnotetext{
* Corresponding author.

Email address:ntha2009@yahoo.com
}

https//doi.org/ 10.25073/2588-1124/vnumap.4417 
where $\mathrm{A}(),. \mathrm{B}($.$) are continuous matrix functions defined on \mathbf{T} \cap[a, \infty)$ valued in $\mathbb{R}^{n \times n}$. The leading term $A_{\sigma}(t)$ (rd-continuous) is supposed to be singular for all $t \geq a$. If the system (1.1) is subjected to an outer force q, then it becomes

$$
A_{\sigma}(t) y^{\Delta}(t)=B(t) y(t)+q(t), \quad t \geq a, t \in \mathbf{T},
$$

If $\mathbf{T}=\mathbb{R}$ then the IDE (1.2) becomes a time-varying differential-algebraic equation and if $\mathbf{T}=\mathbb{Z}$ then it is a time-varying implicit difference equation. The differential-algebraic equations (DAEs) are the mathematical models arising in various applications, such as multibody mechanics, electrical circuits, prescribed path control, chemical engineering, etc., see [2, 3]. Similarly, the implicit difference equations also occur in the different fields, such as population dynamics, economics, systems and control theory, and numerical analysis, etc., see $[4,5]$. Therefore, it is very meaningful to have an equation combining these equations. Indeed, this can be done by the theory of dynamic systems on an arbitrary time scale. This theory has been found promising because it demonstrates the interplay between the theories of continuous time and discrete time systems, see, e.g. [6, 7]. By using this theory, the IDE can be considered as a unified and connected form between the time-varying DAE and the time-varying implicit difference equation. Thus, they play an important role in mathematical modeling with many applications.

On the basis of the above discussion, it is worth considering the robust stability of these equations. To study that, the index notion, which plays a key role in the qualitative theory and in the numerical analysis of IDEs, should be taken into consideration in the robust stability analysis, (see [8, 9]). For the stability theory of time-varying linear DAEs, a few contributions are available (see [10, 11]). To develop from DAEs to IDEs, the results of the paper are to derive some characterizations for the robust stability of IDEs subjected to Lipschitz perturbations.

The paper is organized as follows. In the next section we recall some basic notions and preliminary results on time scales and deal with the solvability of IDEs. In Section 3, we prove that if the system (1.1) is exponentially stable, then under small Lipschitz perturbations, the system (1.1) is still exponentially stable. In the end of this paper, we also give the example to illustrate the theorem in Section 3.

\section{Solvability of implicit linear dynamic equations on time scales}

In recent years, to unify continuous and discrete analysis or to describe the processing of numerical calculation with non-constant steps, a new theory was born and is more and more extensively concerned, that is the theory of the analysis on time scales, which was introduced by Stefan Hilger 1988 (see [12]). A time scale is a nonempty closed subset of the real numbers $\mathbb{R}$, enclosed with the topology inherited from the standard topology on $\mathbb{R}$. We usually denote it by the symbol $\boldsymbol{T}$. We define the forward jump operator $\sigma(t)=\inf \{s \in \mathbf{T}: s>t\}$ and the graininess $\mu(t)=\sigma(t)-t$. Simi;ar;y, the backward operator is defined as $\rho(t)=\sup \{s \in \mathrm{T}: s<t\}$ and the backward graininess is $v(t)=t-\rho(t)$. A point $t \in \mathbf{T}$ is said to be right-dense if $\sigma(t)=t$, rightscattered if $\sigma(t)>t$, left-dense if $\rho(t)=t$, left-scattered if $\rho(t)<t$ and isolated if $\mathrm{t}$ is simultaneously right-scattered and left-scattered.

A function $f$ defined on $T$ valuated in $\mathbb{R}$ is regulated if there exist the left-sided limit at every left-dense point and right-sided limit at every right-dense point. A regulated function $f$ is called $r d$ - 
continuous if it is continuous at every right-dense point, and ld-continuousif it is continuous at every left-dense point. The set of rd-continuous functions defined on the interval $J$ valued in $X$ will be denoted by $C_{r d}(J, X)$. A function $f$ from $\mathbf{T}$ to $\mathbb{R}$ is regressive (resp., positively regressive) if for every $t \in \mathbf{T}$, then $1+\mu(t) \neq 0$ (resp., $1+\mu(t)>0$ ). We denote by $\mathfrak{R}=\mathfrak{R}(\mathbf{T}, \mathbb{R})\left(\right.$ resp., $\mathfrak{R}^{+}=\mathfrak{R}^{+}(\mathbf{T}, \mathbb{R})$ ) the set (resp., positively regressive) regressive functions, and $C_{r d} \mathfrak{R}(\mathbf{T}, \mathbb{R})$ (resp., $C_{r d} \mathfrak{R}^{+}(\mathbf{T}, \mathbb{R})$ ) the set of rd-continuous (resp., positively regressive) regressive functions from $\mathbf{T}$ to $\mathbb{R}$. It is easy to verify that, for all $p, q \in \mathfrak{R}, p \oplus q, p \Theta q \in \mathfrak{R}$. Element $(\Theta q)($.$) is called the inverse element of element$ $q(.) \in \mathfrak{R}$.Hence, the set $\mathfrak{R}(\mathbf{T}, \mathbb{R})$ with the calculation $\oplus$ forms an Abelian group.

Definition 1.1 ([12]). (Delta Derivative). A function $\varphi: \mathbf{T} \rightarrow \mathbb{R}^{d}$ is called delta differentiable at $\mathrm{t}$ if there exists a vector $\varphi^{\Delta}(t)$ such that for all $\varepsilon>0$,

$$
\varphi(\sigma(t))-\varphi(s)(\sigma(t)-s) \leq \varepsilon|\sigma(t)-s|,
$$

for all $s \in(t-\delta, t+\delta) \cap \mathbf{T}$ and for some $\delta>0$. The vector $\varphi^{\Delta}(t)$ is called the deltaderivative of $\varphi$ at t.

Theorem 1.2 ([12]). If $p$ is regressive and fix $t_{0} \in \mathbf{T}$, then the only solution of the initial value problem

on $\mathbf{T}$ is definedby $e_{p}\left(t, t_{0}\right)$, where

$$
y^{\Delta}(t)=p(t), \quad y\left(t_{0}\right)=1
$$

$$
e_{p}\left(t, t_{0}\right)=\exp \left\{\int_{t_{0}}^{t} \lim _{u \rightarrow \mu(\tau)} \frac{\operatorname{Ln}(1+u p(\tau))}{u} \Delta \tau\right\} .
$$

The further details on the analysis on time scales can be referred to [12].

On the language of analysis on time scales, the linear algebraic differentiable equation

$$
A(t) y^{\prime}(t)=B(t) y(t)+q(t), \quad t \geq a,
$$

and the implicit difference equation

$$
A_{n+1}(t) y(n+1)=B(n) y(n)+q(n), \quad n \geq a,
$$

can be unified under the form, called the time-varying linear IDE on time scale $\mathbf{T}$

$$
A_{\sigma}(t) y^{\Delta}(t)=B(t) y(t)+q(t), \quad t \geq a,
$$

where A, B are continuous matrix functions as in Section 1, i.e. $\operatorname{rank} \mathrm{A}(t)=r, 1 \leq r<n$, for all $t \in \mathbf{T}_{a}$ and $q$ is a continuous function defined on $\mathbf{T}_{a}$, valued in $\mathbb{R}^{n}$.

Firstly, we consider the solvability of the IDE (2.1). Assume that $\operatorname{Ker} A(t)$ is smooth in the sense there exists an continuously differential projector $Q(t)$ onto $\operatorname{Ker} A(t)$, i.e., $Q \in C^{1}\left(\mathbf{T}_{a}, \mathbb{R}^{n \times n}\right)$. Set $P=I-Q$, then $P(t)$ is a projector along $\operatorname{Ker} A(t)$. With these notations, the system (2.4) can be rewritten into the form

$$
A_{\sigma}(t)(P y)^{\Delta}(t)=\bar{B}(t) y(t)+q(t)
$$

where $\bar{B}=B+A_{\sigma} P^{\Delta} \in L_{\infty}^{l o c}\left(\mathbf{T}, \mathbb{R}^{n \times n}\right)$. 
Let $\mathrm{H}$ be a continuous function defined on $\mathbf{T}_{a}$, taking values in $G l\left(\mathbb{R}^{n}\right)$ such that $\left.H\right|_{\text {KerA }_{\sigma}}$ is an isomorphism between $\operatorname{Ker} A_{\sigma}(t)$ and $\operatorname{Ker} A(t)$. Define $G=A_{\sigma}-\bar{B} H Q_{\sigma}$ and $S=\left\{y: \operatorname{B} y \in \operatorname{Im} A_{\sigma}\right\}$.

Lemma 2.2 The following assertions are equivalent
a) $S \cap \operatorname{Ker} A=\{0\}$;
b) The matrix $G=A_{\sigma}-\bar{B} H Q_{\sigma}$ is nonsingular;
c) $\mathbb{R}^{n}=S \oplus K \operatorname{er} A$;

Proof See [13, Lemma 2.1].

Lemma 2.3 Suppose that the matrix $\mathrm{G}$ is nonsingular. Then, there hold the following relations:
a) $P_{\sigma}=G^{-1} A_{\sigma}$;
b) $G^{-1} \bar{B} H Q_{\sigma}=-Q_{\sigma}$;
c) $\tilde{Q}=-Q_{\sigma} G^{-1} \bar{B}$ is the projector onto KerA along $\mathrm{S}$;
If $\hat{Q}$ is a projector onto KerA then
d) $P_{\sigma} G^{-1} \bar{B}=P_{\sigma} G^{-1} \bar{B} \hat{P}$, and $Q_{\sigma} G^{-1} \bar{B}=Q_{\sigma} G^{-1} \bar{B} \hat{P}-H^{-1} \hat{Q}$, with $\hat{P}=I-\hat{Q}$.
e) $P_{\sigma} G^{-1}, H Q_{\sigma} G^{-1}$ do not depend on the choice of $\mathrm{T}$ and $\mathrm{Q}$.

Proof See [13, Lemma 2.2].

Definition 2.4 (see also [6, Section 1.2]) The DAE (2.1) is said to be index-1 tractable on $\mathbf{T}$ if $G(t)$ is invertible for almost every $t \in \mathbf{T}$.

Now let (2.1) be index-1. Multiplying both sides of (2.2) with $P_{\sigma} G^{-1}, Q_{\sigma} G^{-1}$ respectively, we can decouple the index-1 equation (2.1) into the system

$$
\left\{\begin{array}{l}
(P y)^{\Delta}=\left(P^{\Delta}+P_{\sigma} G^{-1} \bar{B}\right) P y+P_{\sigma} G^{-1} q \\
Q y=H Q_{\sigma} G^{-1} \bar{B} P y+H Q_{\sigma} G^{-1} q .
\end{array}\right.
$$

Denote $u=P y, v=Q y$, we obtain

$$
\left\{\begin{array}{l}
u^{\Delta}=\left(P^{\Delta}+P_{\sigma} G^{-1} \bar{B}\right) u+P_{\sigma} G^{-1} q \\
v=H Q_{\sigma} G^{-1} \bar{B} u+H Q_{\sigma} G^{-1} q .
\end{array}\right.
$$

Multiplying both sides of (2.3) with $Q_{\sigma}$ yields $Q_{\sigma} u^{\Delta}=Q_{\sigma} P^{\Delta} u$ which implies that $(Q u)^{\Delta}=Q^{\Delta} Q u$. Hence, if $Q\left(t_{0}\right) u\left(t_{0}\right)=0$ then $Q(t) u(t)=0$ for all $t \in \mathbf{T}_{t_{0}}$, i.e. the equation (2.3) has the invariant property in the sense that every solution starting in $\operatorname{Im} P\left(t_{0}\right)$ remains in $\operatorname{Im} P(t)$ for all $\mathrm{t}$.

We consider the homogeneous case $q(t)=0$,

$$
A_{\sigma}(t) y^{\Delta}(t)=B(t) y(t), \quad t \geq a,
$$

and construct the Cauchy operator generated by (2.5). Let $\Phi_{0}(t, s)$ denote the Cauchy operator generated by the equation (2.3), i.e.,

$$
\Phi_{0}^{\Delta}(t, s)=\left(P^{\Delta}(t)+P_{\sigma}(t) G^{-1} \bar{B}(t)\right) \Phi_{0}(t, s) \text {, and } \Phi_{0}(s, s)=I .
$$


Then, the Cauchy operator generated by system (2.5) is defined by

$$
\left\{\begin{array}{l}
A_{\sigma}(t) \Phi^{\Delta}(t, s)=B(t) \Phi(t, s), \\
P(s)(\Phi(s, s)-I)=0
\end{array}\right.
$$

for all $t \geq s \geq a$ and it can be given as follows:

$$
\Phi(t, s)=\left(I+H Q_{\sigma} G^{-1} \bar{B}\right) \Phi_{0}(t, s) P(s)=\tilde{P}(t) \Phi_{0}(t, s) P(s),
$$

where $\tilde{P}(t)=\left(I+H Q_{\sigma} G^{-1} \bar{B}\right)$.

$$
P(t) \Phi(t, s)=P(t) \tilde{P}(t) \Phi_{0}(t, s) P(s)=P(t) \Phi_{0}(t, s) P(s)=\Phi_{0}(t, s) P(s) .
$$

By the arguments used in [6, Section 1.2], the unique solution of the initial value problem for (2.1) with the initial condition

$$
P\left(t_{0}\right)\left(y\left(t_{0}\right)-y_{0}\right)=0, \quad t \geq t_{0},
$$

By the variation of constants formula, we have the unique solution of Equation (2.1) defined by

$$
y(t)=\Phi\left(t, t_{0}\right) P\left(t_{0}\right) y_{0}+\int_{t_{0}}^{t} \Phi(t, \sigma(s)) P_{\sigma} G^{-1} q(s) d s+H Q_{\sigma} G^{-1} q(t) .
$$

\section{Robust stability of perturbed implicit dynamic equations on time scales}

In the following, we suppose that, there exists a bounded differential projector $Q(t)$ onto $\operatorname{KerA}(t)$

and $N_{0}=\sup _{t \in \mathbf{T}_{a}}\|P(t)\| . \quad$ We consider the system (2.1) with perturbations of the form $q(t)=f(t, y(t))$ where $f$ is a certain function defined on $\mathbf{T}_{a} \rightarrow \mathbb{R}^{n}$. Then, the equation (2.1) becomes

$$
A_{\sigma}(t) y^{\Delta}(t)=B(t) y(t)+f(t, y(t)), \quad t \geq a .
$$

Let $f(t, 0)=0$ for all $t \in \mathbf{T}_{a}$, which implies that Equation (3.1) has the trivial solution $x(t) \equiv 0$. As before, denoting $\mathrm{u}=\mathrm{Py}$ and $\mathrm{v}=\mathrm{Qy}$ comes to

$$
\begin{aligned}
& u^{\Delta}=\left(P^{\Delta}+P_{\sigma} G^{-1} \bar{B}(t)\right) u+P_{\sigma} G^{-1} f(t, u+v), \\
& v=H Q_{\sigma} G^{-1} \bar{B} u+H Q_{\sigma} G^{-1} f(t, u+v) .
\end{aligned}
$$

Assume that $H Q_{\sigma} G^{-1} f(t,$.$) is Lipschitz continuous with the Lipschitz coefficient \gamma_{t}<1$, i.e.,

$$
\left\|H Q_{\sigma} G^{-1} f(t, y)-H Q_{\sigma} G^{-1} f(t, x)\right\|<\gamma_{t}\|y-x\|, \text { for all } t \geq a .
$$

Since $H Q_{\sigma} G^{-1}$ does not depend on the choice of $\mathrm{H}$ and Q, so does the Lipschitz property of $H Q_{\sigma} G^{-1} f$. Fix $u \in \mathbb{R}^{m}$ and choose $t \in \mathbf{T}_{a}$, we consider a mapping $\Gamma_{t}: \operatorname{Im} Q(t) \rightarrow \operatorname{Im} Q(t)$ defined by

$$
\Gamma_{t}(v)=H(t) Q_{\sigma}(t) G^{-1}(t) \bar{B}(t) u+H(t) Q_{\sigma}(t) G^{-1}(t) f(t, u+v) .
$$

It is easy to see that

$$
\left\|\Gamma_{t}(u)-\Gamma_{t}(v)\right\|<\gamma_{t}\|u-v\| \text {, for any for all } v, v^{\prime} \in \operatorname{Im} Q .
$$

Since $\gamma_{t}<1, \Gamma_{t}$ is a contractive mapping. Hence, by the Fixed Point Theorem, there exists a mapping $g_{t}: \operatorname{Im} P(t) \rightarrow \operatorname{Im} Q(t)$ satisfying 


$$
g_{t}(u)=H(t) Q_{\sigma}(t) G^{-1}(t) \bar{B}(t) u+H(t) Q_{\sigma}(t) G^{-1}(t) f\left(t, u+g_{t}(u)\right) .
$$

On the other hand,

$$
\left\|g_{t}(u)-g_{t}\left(u^{\prime}\right)\right\| \leq \beta_{t}\left\|u-u^{\prime}\right\|+\gamma_{t}\left(\left\|u-u^{\prime}\right\|+g_{t}(u)-g_{t}\left(u^{\prime}\right)\right),
$$

where $\beta_{t}=\left\|H(t) Q_{\sigma}(t) G^{-1}(t) \bar{B}(t)\right\|$. This deduces

$$
\left\|g_{t}(u)-g_{t}\left(u^{\prime}\right)\right\| \leq \frac{\gamma_{t}+\beta_{t}}{1-\gamma_{t}}\left\|u-u^{\prime}\right\| .
$$

Thus, $g_{t}$ is Lipschitz continuous with the Lipschitz coefficient $L_{t}=\frac{\gamma_{t}+\beta_{t}}{1-\gamma_{t}}$. Substituting $v=g_{t}(u)$ into (3.2) obtains

$$
u^{\Delta}=\left(P^{\Delta}+P_{\sigma} G^{-1} \bar{B}(t)\right) u+P_{\sigma} G^{-1} f\left(t, u+g_{t}(u)\right) .
$$

Suppose that (3.5) is solvable. Then we find $u(t)$ from Equation (3.5) and get the solution of (3.1) by $y(t)=u(t)+g_{t}(u(t)), t \in \mathbf{T}_{a}$.

Definition 3.1. 1. The solutions of IDE (2.5) are said to be bounded if $\sup \left\{\left\|y\left(t, t_{0}, x_{0}\right)\right\|, t \in \mathbf{T}_{t_{0}}\right\}<\infty$ for all $y_{0} \in \mathbb{R}^{n}$. The solutions of (2.5) are uniformly bounded if $\sup \left\{\left\|y\left(t, t_{0}, x_{0}\right)\right\|, t, t_{0} \in \mathbf{T}_{a}\right\}<\infty$ for all $y_{0} \in \mathbb{R}^{n}$.

2. The IDE (2.5) is said to be exponential stable if there exist the positive numbers $M>0, \alpha>0$ such that $-\alpha \in \mathfrak{R}_{+}$and

$$
\left\|y\left(t, t_{0}, x_{0}\right)\right\| \leq M e_{-\alpha}\left(t, t_{0}\right)\left\|\tilde{P}\left(t_{0}\right) y_{0}\right\|, \quad t \geq t_{0} \geq a, \quad \mathrm{y}_{0} \in \mathbb{R}^{n} .
$$

Following the classical way, we see that exponential stabilily and uniformly stability of IDE are characterized in term of its transition operator as the follows:

Theorem 3.2. a. The IDE (2.5) is uniformly stable if and only if there exists the positive number $M_{0}$ such that

$$
\|\Phi(t, s)\| \leq M_{0}, \quad t \geq s \geq a .
$$

b. The DAE (2.5) is exponentially stable if and only if there exists the positive numbers $M_{1}>0, \alpha>0$ such that $-\alpha \in \mathfrak{R}_{+}$and

$$
\|\Phi(t, s)\| \leq M_{1} e_{-\alpha}(t, s), \quad t \geq s \geq a .
$$

Proof See [14].

Lemma 3.3 If the index-1 tracable (2.6) is stable, there is a positive numbers $M_{0}>0$ such that

$$
\left\|\Phi_{0}(t, s) P(s)\right\| \leq K_{0} .
$$

If it is exponentially stable, then there exists the positive numbers $M>0$ such that

$$
\left\|\Phi_{0}(t, s) P(s)\right\| \leq M e_{-\alpha}(t, s), \mathrm{t} \geq s \geq a .
$$

Proof From the formula (2.7) we see that 


$$
P(t) \Phi(t, s)=P(t) \tilde{P}(t) \Phi_{0}(t, s) P(s)=P(t) \Phi_{0}(t, s) P(s)=\Phi_{0}(t, s) P(s) .
$$

With the boundedness of the projector $P(t)$ as in above assumption, we have

$$
\left\|\Phi_{0}(t, s) P(s)\right\|=\|P(t) \Phi(t, s)\| \leq \sup _{t \in \mathbf{T}_{a}}\|P(t)\|\|\Phi(t, s)\| \leq N_{0}\|\Phi(t, s)\| .
$$

Thus, from the inequality(3.6), there exists a constant $K_{0}=N_{0} M>0$ such that

$$
\left\|\Phi_{0}(t, s) P(s)\right\| \leq K_{0} .
$$

Respectively, from the inequality (3.7), there is a constant $M=M_{1} \sup _{t \in \mathbf{T}_{a}}\|P(t)\|>0$ such that

$$
\left\|\Phi_{0}(t, s) P(s)\right\| \leq M e_{-\alpha}(t, s), \mathrm{t} \geq s \geq a .
$$

We have the proof.

Now, we are in the positionto consider the uniform stability of (2.1) under small perturbations.

For the uniform stability, we have the following result.

Theorem 3.4 Assume that the equation (2.5) is index-1, uniformly stable and

a) $L=\sup _{t \in \mathbf{T}_{a}} L_{t}<\infty$.

b) The function $P_{\sigma}(t) G^{-1}(t) f(t, y)$ is Lipschitz continuous with the Lipschitz coefficient $k_{t}$.

Further, $M_{0} \int_{a}^{\infty} k_{t}\left(1+L_{t}\right) \Delta t=N<\infty$.

Then, the system (3.1) is uniformly stable, i.e., there exists a constant $M_{1}>0$ such that the solution $\mathrm{x}(\cdot)$ of (3.1) satisfies

$$
\|y(t)\| \leq M_{1}\|y(s)\|, \quad \forall t>s \geq a .
$$

Proof By using the constant-variation formula (2.8), for all $t>s \geq a$, we have

$$
u(t)=\Phi_{0}\left(t, t_{0}\right) u(s)+\int_{s}^{t} \Phi_{0}(t, \sigma(\tau)) P_{\sigma} G^{-1} f\left(\tau, u(\tau)+g_{\tau}(u(\tau))\right) \Delta \tau .
$$

Therefore,

$$
\begin{gathered}
\|u(t)\|=\left\|\Phi_{0}\left(t, t_{0}\right) u(s)\right\|+\int_{s}^{t}\left\|\Phi_{0}(t, \sigma(\tau))\right\|\left\|P_{\sigma} G^{-1} f\left(\tau, u(\tau)+g_{\tau}(u(\tau))\right)\right\| \Delta \tau . \\
\leq M_{0}\|u(s)\|+M_{0} \int_{s}^{t} k_{t}\left(1+L_{t}\right)\|u(\tau)\| \Delta \tau .
\end{gathered}
$$

By using Gronwall-Bellman inequality, we get $\|u(t)\| \leq M_{0}\|u(s)\| e_{p(t)}(t, s)$, where $p(t)=M_{0} k_{t}\left(1+L_{t}\right)$, Since $p$ is positive,

$$
e_{p}(t, s) \leq \exp \left(\int_{s}^{t} p(\tau) d \tau\right) \leq e^{N} .
$$

Therefore $\|u(t)\| \leq M_{0} e^{N}\|u(s)\|$. On the other hand

$$
\|u(s)\|=\|P(s) u(s)\|=\|P(s) x(s)\| \leq K_{0}\|x(s)\|,
$$


and

$$
\|y(t)\| \leq\|u(t)\|+\|g(u(t))\| \leq(1+L)\|u(t)\| \leq(1+L) M_{0} e^{N}\|u(s)\| \leq M_{1}\|y(s)\|,
$$

where $M_{1}=(1+L) M_{0} K_{0} e^{N}$. The proof is complete.

Theorem 3.5 If the equation (2.5) is index-1, exponential stable and

a. $\quad L=\sup _{t \in \mathbf{T}_{a}} L_{t}<\infty$.

b. $\quad \limsup _{t \rightarrow \infty} k_{t}\left(1+L_{t}\right)=\delta<\frac{\alpha}{M}$, with $\alpha, M$ is defined by Definition 3.1.

Then, there exist constants $\mathrm{K}>0$ and $-\alpha_{1} \in \mathfrak{R}^{+}$such that

$$
\|y(t)\| \leq K e^{-\alpha_{1}(t-s)}\|y(s)\|, \quad \forall t \geq s \geq t_{0} .
$$

for every solution $\mathrm{x}(\cdot)$ of $(3.1)$. That is, the perturbed equation (3.1) preserves the exponential stability. Proof. Let $\varepsilon$ be a positive number such that $\delta+\varepsilon<\frac{\alpha}{L M}$. Then, by the second assumption, there exists $T_{0}>t_{0}$ such that

$$
k_{t}\left(1+L_{t}\right)<\delta+\varepsilon, \quad \forall t>T_{0} .
$$

By the continuity of the solutions of (3.5) on the initial condition we can find a constant $M_{T_{0}}>0$ (where $M_{T_{0}}$ depends only on $T_{0}$ ) such that

$$
\|u(t)\| \leq M_{T_{0}}\|u(s)\|, \quad \text { for all } t_{0} \leq s \leq t \leq T_{0} .
$$

First, we consider the case $t>T_{0}>s \geq t_{0}$. Then, follow the estimations (3.9) and (3.10), we get

$$
\begin{aligned}
\|u(t)\| \leq & \left\|\Phi_{0}\left(t, T_{0}\right) u\left(T_{0}\right)\right\|+\int_{T_{0}}^{t}\left\|\Phi_{0}(t, \sigma(\tau)) P_{\sigma} \tilde{G}^{-1} f\left(\tau, u(\tau)+g_{\tau}(u(\tau))\right)\right\| \Delta \tau \\
& \leq M e_{-\alpha}\left(t, T_{0}\right)\left\|u\left(T_{0}\right)\right\|+\int_{T_{0}}^{t} e_{-\alpha}(t, \sigma(\tau)) k_{\tau}\left(1+L_{\tau}\right)\|\tau(\tau)\| \Delta \tau \\
& \leq M e_{-\alpha}\left(t, T_{0}\right)\left\|u\left(T_{0}\right)\right\|+M(\delta+\varepsilon) \int_{T_{0}}^{t} \frac{e_{-\alpha}(t, \tau)}{1-\alpha \mu(\tau)}\|u(\rho)\| \Delta \tau .
\end{aligned}
$$

Multiplying both sides of the above inequality with $\frac{1}{e_{-\alpha}\left(t, T_{0}\right)}$ yields

$$
\frac{\|u(t)\|}{e_{-\alpha}\left(t, T_{0}\right)} \leq M\left\|u_{T_{0}}\right\|+M(\delta+\varepsilon) \int_{T_{0}}^{t} \frac{\|u(\tau)\|}{(1-\alpha \mu(\tau)) e_{-\alpha}\left(\tau, T_{0}\right)} \Delta \tau .
$$

By using Gronwall-Bellman inequality, we obtain

$$
\frac{\|u(t)\|}{e_{-\alpha}\left(t, T_{0}\right)} \leq M\left\|u_{T_{0}}\right\| e_{\frac{M(\delta+\varepsilon)}{1-\alpha \mu(t)}}\left(t, T_{0}\right)
$$

Therefore, 


$$
\|u(t)\| \leq M\left\|u_{T_{0}}\right\| e_{-\alpha \oplus \frac{M(\delta+\varepsilon)}{1-\alpha \mu(t)}}\left(t, T_{0}\right)=M\left\|u_{T_{0}}\right\| e_{-\alpha+M(\delta+\varepsilon)}\left(t, T_{0}\right) .
$$

Let $\alpha_{1}=-\alpha+M(\delta+\varepsilon)$, since $-\alpha \in \mathfrak{R}^{+}$so is $-\alpha_{1} \in \mathfrak{R}^{+}$. Therefore

$$
\|u(t)\| \leq \frac{M\left\|u_{T_{0}}\right\|}{e_{-\alpha_{1}}\left(T_{0}, s\right)} e_{-\alpha_{1}}(t, s) \leq M e_{\Theta-\alpha_{1}}\left(T_{0}, t_{0}\right) e_{-\alpha_{1}}(t, s)\left\|u\left(T_{0}\right)\right\| \leq M M_{T_{0}} e_{\Theta-\alpha_{1}}\left(T_{0}, t_{0}\right) e_{-\alpha_{1}}(t, s)\|u(s)\| .
$$

Thus, $\|u(t)\| \leq K_{1} e_{-\alpha_{1}}(t, s)\|u(s)\|$, where $K_{1}=M M_{T_{0}} e_{\Theta-\alpha_{1}}\left(T_{0}, t_{0}\right)$.

In the case $t>s \geq T_{0}$, it follows from the estimate $\left\|P_{\sigma} G^{-1}(t)\left(\tau, u+g_{\tau}(u)\right)\right\| \leq\left(\delta+\varepsilon_{0}\right)\|u\|$ holds for all $\tau \geq s$. Similarly, we have

$$
\|u(t)\| \leq M_{0} e_{-\alpha_{1}}(t, s)\|u(s)\| .
$$

For the remaining case $t_{0} \leq s<t \leq T_{0}$, with $\alpha_{1}>0$ defined above, we have

$$
\|u(t)\| \leq M_{T_{0}}\|u(s)\|=M_{T_{0}} e_{\alpha_{1}}\left(T_{0}, t_{0}\right) e_{-\alpha_{1}}(t, s)\|u(s)\| .
$$

Put $K_{2}=\max \left\{K_{1}, c M, M_{T_{0}} e_{\alpha_{1}}\left(T_{0}, t_{0}\right)\right\}$, we get

$$
\|u(t)\| \leq K_{2} e_{-\alpha_{1}}(t, s)\|u(s)\| .
$$

Paying attention to (3.6) gets

$$
\|y(t)\| \leq K e_{-\alpha_{1}}(t, s)\|y(s)\|,
$$

where $K K=K_{0} K_{2}(1+L)$. The proof is completed.

Example 3.6 Let the time scale $\mathbf{T}=\cup_{n=0}^{\infty}[2 n, 2 n+1]$. We see that $\sigma(t)=0$ if $t \in[2 n, 2 n+1)$ and $\sigma(t)=1$ if $t=2 n+1$. Consider the perturbed equation (3.1) on $\mathbf{T}$ with

$$
E=\left(\begin{array}{ccc}
1 & 1+t & 0 \\
0 & -1-t & 0 \\
1 & 0 & 0
\end{array}\right), \quad A=\left(\begin{array}{ccc}
-2+\cos \pi t & -1+t & 0 \\
0 & t & 0 \\
-2+\cos \pi t & 1 & 3+t
\end{array}\right), \quad f(t, x)=\left(\begin{array}{c}
\sin \left(x_{1}+x_{2}+x_{3}\right) \\
x_{2} \\
x_{3}
\end{array}\right) .
$$

where $x=\left(x_{1}, x_{2}, x_{3}\right) \in \mathbb{R}^{3}$. LetH=I, it is easily to compute that we have

$$
\begin{aligned}
A & =\left(\begin{array}{lll}
0 & 0 & 0 \\
0 & 0 & 0 \\
0 & 0 & 1
\end{array}\right), \quad Q_{\sigma} G^{-1} B=Q, \quad \bar{B}=B, \quad Q_{\sigma} G^{-1} f=k\left(\begin{array}{c}
0 \\
0 \\
\frac{-\sin \left(x_{1}+x_{2}+x_{3}\right)-x_{2}+x_{3}}{1+t}
\end{array}\right), \text { for all } t \in \mathbf{T} . \\
& \text { If } t \in[2 n, 2 n+1): P_{\sigma} G^{-1} B=\left(\begin{array}{ccc}
-2+\cos \pi t & 1 & 0 \\
0 & -\frac{t}{1+t} & 0 \\
0 & 0 & 0
\end{array}\right), \quad P_{\sigma} G^{-1} f=\left(\begin{array}{c}
\sin \left(x_{1}+x_{2}+x_{3}\right)+x_{2} \\
-\frac{x_{2}}{1+t} \\
0
\end{array}\right) .
\end{aligned}
$$


If $t=2 n+1$, we have $\quad P_{\sigma} G^{-1} B=\left(\begin{array}{ccc}-2+\cos \pi t & 1 & 0 \\ 0 & -\frac{t}{2+t} & 0 \\ 0 & 0 & 0\end{array}\right), \quad P_{\sigma} G^{-1} f=k\left(\begin{array}{c}\sin \left(x_{1}+x_{2}+x_{3}\right)+x_{2} \\ -\frac{x_{2}}{2+t} \\ 0\end{array}\right)$.

Let $s \in[2 m, 2 m+1), t \in[2 n, 2 n+1), m<n$. Asumme that $y(t, s)=\left(y_{1}, y_{2}, y_{3}\right)$ is the solution of the homogeneous equation (2.6) with the initial condition $y(s, s)=\left(y_{1}^{0}, y_{2}^{0}, y_{3}^{0}\right)=y^{0}$ given by $y_{3} \equiv 0$ and

$$
y_{2}(t, s)=y_{2}^{0} e^{-(t-s)-m+n} \frac{1+t}{1+s} \frac{2 m+2}{2 n+1} \prod_{k=m+1}^{n-1} \frac{2+2 k}{1+2 k} \prod_{k=m}^{n-1} \frac{2}{3+2 k}, \quad y_{1}(t, s)=e_{-2+\cos (\pi \cdot)}(t, s)\left(y_{1}^{0}+\int_{0}^{t} \frac{y_{2}(\tau, s)}{e_{-2+\cos (\pi .)}(\tau, s)} \Delta \tau\right) \text {. }
$$

By the caculating on time scale we obtain

$$
e_{-2+\cos (\pi .)}(t, s)=e^{-2[(t-s)+m-n]+\frac{\sin \pi t-\sin \pi s}{\pi}}(-2)^{n-m},
$$

and $\left|y_{2}(t, s)\right| \leq \frac{100}{9}\left|y_{2}^{0}\right| e^{-(t-s)+n-m}\left(\frac{2}{5}\right)^{n-m}<15\left|y_{2}^{0}\right| e_{-0.7}(t, s)$.

Moreover, let $\alpha>0$ be a unique solution of the equation $x-\ln (1-x)-2+\ln 2=0$. It is clear that $0.5<\alpha<0.6$, and

$$
\frac{1}{K} e_{-\alpha}(t, s) \leq\left|e_{-2+\cos (\pi .)}(t, s)\right| \leq K e_{-\alpha}(t, s), \text { with } K=e^{2-\alpha+\frac{1}{\pi}}
$$

Since

$$
\begin{aligned}
\frac{\left|y_{2}(t, s)\right|}{\left|e_{-2+\cos (\pi .)}(\tau, s)\right|} \leq & \frac{100}{9}\left|y_{2}^{0}\right| e^{-(t-s)+n-m}\left(\frac{2}{5}\right)^{n-m} e^{2[(t-s)+m-n]-\frac{\sin \pi t-\sin \pi s}{\pi}}\left(\frac{1}{2}\right)^{n-m} \\
& \leq \frac{100}{9}\left|y_{2}^{0}\right| e^{t-s+m-n+\frac{1}{\pi}}\left(\frac{1}{5}\right)^{n-m} \leq \frac{100}{9}\left|y_{2}^{0}\right| e_{-0.3}(t, s) .
\end{aligned}
$$

It follows that

$$
\int_{0}^{\infty} \frac{\left|y_{2}(\tau, s)\right|}{\left|e_{-2+\cos (\pi .)}(\tau, s)\right|} \leq \frac{100}{9}\left|y_{2}^{0}\right| \int_{0}^{\infty} e_{-0.3}(\tau, s) \leq\left(\frac{10}{3}\right)^{3} e\left|y_{2}^{0}\right|:=c\left|y_{2}^{0}\right| \text {. }
$$

Thus,

$$
\|y(t, s)\| \leq\left(K\left|y_{1}^{0}\right|+(K c+15)\left|y_{2}^{0}\right|\right) e_{-\alpha}(t, s) \leq(K(1+c)+15)\left\|P y^{0}\right\| e_{-\alpha}(t, s), \quad \forall t>s .
$$

This implies that the solution of the IDE (2.6) is asymptotically stable. On the other hand, we have

$$
\gamma_{t}=\frac{k}{1+t}, \quad \beta_{t}=1, \quad k_{t}=4 k, \quad 1+L_{t}=\frac{1+\beta_{t}}{1-\gamma_{t}}, \quad L=1, \quad \lim _{t \rightarrow \infty} k_{t}\left(1+L_{t}\right)=2 k .
$$

Therefore, if $k<\frac{\alpha}{4(K(1+c)+15)}$ then the IDE (3.1) is asymptotically stable by Theorem 3.4. 


\section{Conclusion}

In this paper we have investigated the robust stability for the linear time-varying implicit dynamic equations on time scale. Some characterizations for robust stability of IDEs subjected to Lipschitz perturbations are derived. Many previous results for robust stability of the time-varying ordinary differential and difference equations, the time-varying differential algebraic equations and the timevarying implicit difference equations are also unified and extended.

\section{References}

[1] N.H. Du, L.H. Tien, On the Exponential Stability of Dynamic Equations on Time Scales, J. Math. Anal. Appl. 331 (2007) 1159-1174.

[2] M. Bracke, On stability radii of parametrized linear differential-algebraic systems, Ph.D. Thesis, University of Kaiserslautern, 2000.

[3] P. Kunkel, V. Mehrmann, Differential-Algebraic Equations, Analysis and Numerical Solution. EMS Publishing House, Z"urich, Switzerland, 2006.

[4] V.H. Linh, N.T.T. Nga, D.D. Thuan, Exponential stability and robust stability for linear time-varying singular systems of second-order difference equations, SIAM J. Matrix Anal. Appl. 39 (2018) 204-233.

[5] D.G. Luenberger, Dynamic equations in descriptor form IEEE, Trans. Automat. Control. 22(1977) 312-322.

[6] Z. Bartosiewicz, Linear positive control systems on time scales, Math. Control Signals Syst., 25 (2013) $327-343$.

[7] J.J. DaCunha, J.M. Davis, A unified Floquet theory for discrete, continuous, and hybrid periodic linear systems., J. Differential Equations 251(2011) 2987-3027.

[8] E. Griepentrog, R. M“arz, Differential-algebraic equations and their numerical treatment, Teubner-Texte zur Mathematik, Leibzig 1986.

[9] R. M“arz, Extra-ordinary differential equation attempts to an analysis of differential algebraic system, Progress in Mathematics, 168 (1998) 313-334.

[10] T. Berger, A. Ilchmann, On stability of time-varying linear differential-algebraic equations, International Journal of Control, 86 (2013) 1060-1076.

[11] A.A. Shcheglova, V.F. Chistyakov, Stability of linear differential-algebraic systems, Differential Equations 40(1) (2004) 50-62.

[12] M. Bohner, A. Peterson, Dynamic equations on time scales: An Introduction with Applications, Birkh“auser, Boston, 2001.

[13] N.H. Du, T.K. Duy, V.T. Viet, Degenerate cocycle with index-1 and Lyapunov exponent, Stochatics and Dynamics 7(2)(2007) 229-245.

[14] N.H.Du, N.C. Liem, Linear transformations and Floquet theorem for linear implicit dynamic equations on time scales, Asian-European Journal of Mathematics, 6(1)(2013), 1350004 (21 pages). 